\title{
Identificação dos padrões espectrais da alteração hidrotermal da Mina Uru- guai, Caçapava do Sul (RS), utilizando espectros de reflectância experimen- tal
}

\author{
Raquel B. BINOTTO ${ }^{1,2}$, Dejanira L. SALDANHA ${ }^{1}$, Aline R.A. DIAS ${ }^{3}$, Mônica M. PERROTA ${ }^{4}$ \\ ${ }^{1}$ Programa de Pós Graduação em Sensoriamento Remoto,Universidade Federal do Rio Grande do Sul. Av.Bento Gonçalves, 9500 , \\ CEP 91540-000, Porto Alegre, RS, Brasil.E-mail:raquel.binotto@cprm.gov.br, dejanira.saldanha@ufrgs.br. \\ ${ }^{2}$ Serviço Geológico do Brasil (CPRM), Superintendência Regional de Porto Alegre, Ministério de Minas e Energia. Rua Banco da \\ Província, 105, CEP 90840-030, Porto Alegre, RS, Brasil.E-mail:raquel.binotto@cprm.gov.br. \\ ${ }^{3}$ Votorantim Metais. Rua Luiz Benezato, 500, Polo Industrial Multivias, CEP 13212-161, Jundiaí, SP, Brasil. E-mail: aline.anjos@ \\ vmetais.com.br. \\ ${ }^{4}$ Serviço Geológico do Brasil (CPRM), Superintendência Regional de São Paulo, Ministério de Minas e Energia. Rua Costa, 55, \\ CEP 01304-010, São Paulo, SP, Brasil. E-mail: monica.perrota@cprm.gov.br.
}

Recebido em 01/12/2014. Aceito para publicação em 13/03/2015.

Versão online publicada em 30/04/2015 (www.pesquisasemgeociencias.ufrgs.br)

\begin{abstract}
Resumo - Uma série de análises espectrais foi efetuada em amostras de rochas sedimentares eopaleozoicas afetadas por alteração hidrotermal associada às ocorrências de cobre na Mina Uruguai (Minas do Camaquã, RS). A base de dados contemplou espectros na faixa do visível e infravermelho próximo (350 a $2500 \mathrm{~nm}$ ) obtidos através de leituras em um espectrorradiômetro portátil ASD FieldSpec-3 High-Resolution. A interpretação mineralógica das assinaturas espectrais foi efetuada com o apoio do software The Spectral Geologist (TSG ${ }^{\mathrm{TM}}$ Pro) que permitiu a discriminação das fases minerais presentes por comparação das feições de absorção no espectro analisado com feições similares de minerais de uma biblioteca espectral de referência. A partir da análise espectral das amostras da área de estudo, puderam ser identificados os seguintes minerais principais: caulinita, ilita, clorita, hematita e goetita. As assembleias minerais identificadas se mostraram consistentes com alteração hidrotermal sob condições epitermais de baixo enxofre descrita em estudos anteriores na região. Os resultados obtidos reforçaram o potencial de utilização da espectroscopia de reflectância em programas de exploração mineral, especialmente nas fases iniciais, mesmo em áreas com condições fisiográficas pouco favoráveis.

Palavras-chave: sensoriamento remoto, espectroscopia de reflectância, alteração hidrotermal, cobre.
\end{abstract}

\begin{abstract}
IDENTIFICATION OF HYDROTHERMAL ALTERATION SPECTRAL PATTERNS IN URUGUAI Mine, CAÇAPAVA Do Sul, Rio GRANDE do Sul STATE, BRAZIL, USING EXPERIMENTAL REFLECTANCE SPECTRA. A series of spectral analysis was made with samples of Eopaleozoic sedimentary rocks affected by hydrothermal alteration associated with occurrences of copper in Uruguai Mine (Minas do Camaquã, Rio Grande do Sul State, Brazil). The database included spectral range visible, near infrared and shortwave (350-2500 $\mathrm{nm}$ ) obtained from measurements of portable spectroradiometer ASD FieldSpec-3 High-Resolution. The mineralogical interpretation with spectral signatures was conducted with a support by The Spectral Geologist (TSG ${ }^{\mathrm{TM}}$ Pro) software, which allows a mineral phases discrimination present by comparing the absorption features in the mineral analysis with similar features of a spectral reference library spectrum. Spectral analysis from studied area samples allowed identified these main minerals: kaolinite, illite, chlorite, hematite and goethite. The mineral assemblages identified wereconsistent with hydrothermal alteration under conditions of low sulphur epithermal described in previous studies in this region. The results supported a potential use of reflectance spectroscopy in mineral exploration programs, especially in the early stages, even in areas with little favorable physiographic conditions.
\end{abstract}

Keywords: remote sensing, reflectance spectroscopy, hydrothermal alteration, copper.

\section{Introdução}

A espectroscopia de reflectância na região do visível, infra-vermelho próximo e de ondas curtas
(VNIR e SWIR) tem sido utilizada como um método rápido e não destrutivo na exploração mineral para a identificação de padrões hidrotermais em diferentes depósitos (Hunt \& Ashley, 1979; Herr- 
mann et al., 2001; Yang et al., 2001; Sun et al., 2001; Jones et al., 2005; Yang et al., 2005; Cudahy et al., 2009; Herrmann et al., 2009; Doublier et al., 2010; Laukamp et al., 2011).No Brasil, a concepção, elaboração e operacionalização da Biblioteca Espectral do Serviço Geológico do Brasil, coordenada pela Divisão de Sensoriamento Remoto (DISERE/ CPRM), está em processo de implantação, cujos dados tem sido utilizados em diferentes estudos nas mais diversas aplicações (Perrota, 2004; Perrota et al., 2005, 2012, 2013, 2014; Bergmann et al., 2008, 2009; Justo et al., 2012, 2013).

A abundância, composição e grau de cristalinidade de certos minerais podem ser inferidos pela profundidade, posição e forma de feições de absorção características na região do SWIR (Sonntag et al., 2012).

As curvas espectrais de reflectância dos minerais exibem feições diagnósticas em diferentes comprimentos de onda que permitem sua discriminação e identificação. Estas feições são produzidas por processos eletrônicos ou vibracionais resultantes da interação entre a energia com os átomos/moléculas que formam os minerais. Os espectros dos diferentes minerais são combinados para formar a assinatura espectral de determinada rocha. Os espectros de reflectância dos minerais em comprimentos de onda inferiores a $1000 \mathrm{~nm}$ são dominados por processos envolvendo metais de transição, sendo o Ferro o mais comum na superfície da Terra. Assim, nos espectros das rochas na faixa de 400 a $1000 \mathrm{~nm}$ predominam feições associadas a minerais contendo óxidos de ferro. Entre 1000 e 2500 nm, são características nos espectros dos minerais combinações envolvendo o íon hidroxila $(\mathrm{OH})$, resultando na discriminação de feições espectrais características dos minerais de argila. Os carbonatos também apresentam várias feições espectrais nesta faixa envolvendo combinações entre o Carbono e o Oxigênio (Hook et al., 1994).

Considerando que a estabilidade de cada mineral e das assembleias minerais depende de parâmetros físico-químicos, tais como temperatura, pressão e $\mathrm{pH}$, sua ocorrência associada às diferentes zonas de alteração hidrotermal é indicativa dos processos formadores do depósito, fornecendo informações importantes sobre o tipo e localização do minério.

Por exemplo, em depósitos de cobre pórfiro, filossilicatos hidrotermais como muscovita, ilita e clorita são considerados essenciais na identificação e entendimento das zonas de alteração. A identificação dos diferentes tipos de micas e argilominerais formados em sistemas hidrotermais em amostras de mão utilizando ferramentas comuns (lupas de mão) é difícil. 0 termo "sericita" é comumente utilizado nas campanhas de campo para se referir aos minerais finamente granulares do grupo das micas brancas. Embora o grupo de minerais da clorita possa ser identificado em amostras de mão, tipos composicionais específicos do grupo que distinguem o tipo de alteração também não podem ser identificados através de métodos comumente utilizados no campo (Cohen, 2011).

Neste contexto, no presente artigo é caracterizada espectralmente a alteração hidrotermal associada às ocorrências de cobre na Mina Uruguai, depósito de $\mathrm{Cu}(\mathrm{Au} / \mathrm{Ag})$, cujo modelo genético mais característico é do tipo epitermal de baixo enxofre (Laux et al., 2005), a partir da análise qualitativa e quantitativa de dados de espectroscopia de reflectância. 0 padrão espectral da alteração hidrotermal do depósito estudado poderá ser reconhecido em outros alvos de interesse em estudos futuros na região.

\section{2 Área, materiais e métodos}

A área de estudo está situada na porção centro-sul do Estado do Rio Grande do Sul, no município de Caçapava do Sul, na localidade de Minas do Camaquã, a aproximadamente $350 \mathrm{~km}$ da capital do estado, Porto Alegre, com acesso através da BR153. As coordenadas de referência da Mina Uruguai são $30^{\circ} 54^{\prime} 34^{\prime \prime}$ Sul e 5326'37" Oeste (Fig. 1).

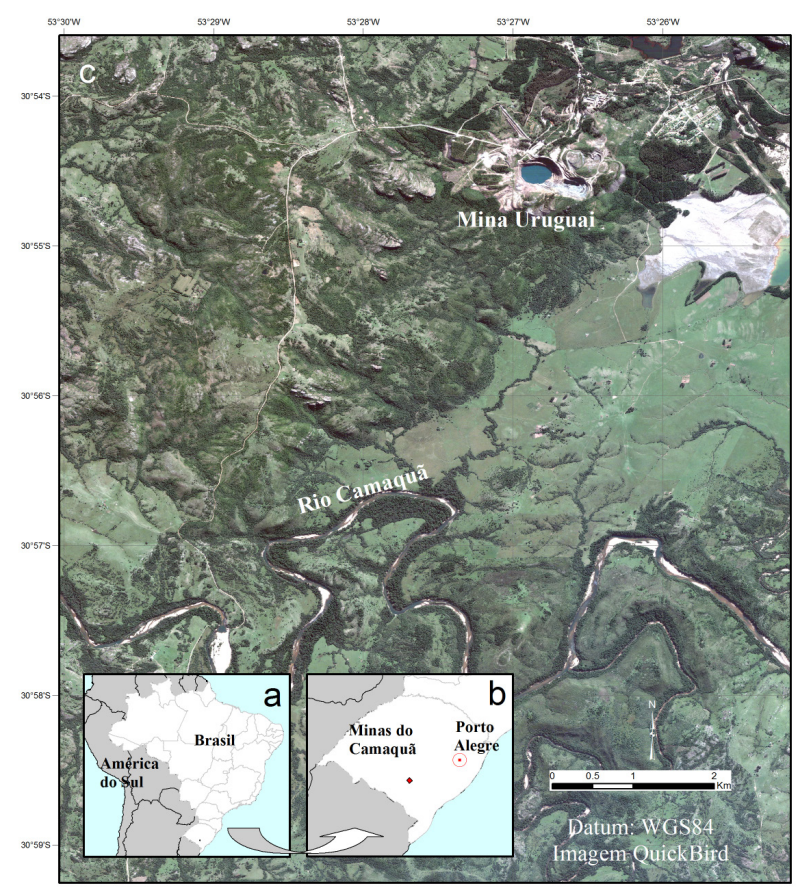

Figura 1. Localização da área de estudo: A) Situação no país; B) Situação no estado do Rio Grande do Sul e em relação à capital, Porto Alegre; C) Mina Uruguai, minas do Camaquã, Rio Grande do Sul, Brasil. 


\subsection{Contexto geológico}

As Minas do Camaquã estão localizadas em um gráben com direção geral nordeste, identificando-se dois sistemas de falhas principais, um regional, com direção nordeste $\left(\mathrm{N} 20^{\circ}-30^{\circ} \mathrm{E}\right)$, responsável pelos limites e compartimentação longitudinal do gráben, e outro controlando as principais estruturas mineralizadas, noroeste $\left(\mathrm{N} 50^{\circ}-70^{\circ} \mathrm{W}\right)$ (Troian, 2009).

No contexto geológico regional, as rochas, na região das minas do Camaquã, inserem-se no Grupo Santa Bárbara (Fambrini, 2003) (Fig.2), de idade eopaleozoica, cujas rochas sedimentares classicamente são relacionadas à Formação Arroio dos Nobres por outros autores (e.g., Ribeiro et al., 1966; Gonzalez \& Teixeira, 1980; Paim et al., 1992), membros Mangueirão e Vargas, sendo as rochas deste último hospedeiras das mineralizações de $\mathrm{Cu}-\mathrm{Pb}-\mathrm{Zn}$.

O Membro Vargas é constituído por um pacote de arenitos e conglomerados, com mergulho em torno de $\mathrm{N} 30^{\circ} \mathrm{E}$, tendo sido subdividido, na área das minas do Camaquã, da base para o topo, em cinco unidades litológicas (Fig. 3): Arenito Inferior, Conglomerado Inferior, Arenito Intermediário, Conglomerado Superior e Arenito Superior. Com exceção do Arenito Superior, as demais unidades apresentam mineralizações (Bettencourt, 1972;
Teixeira et al., 1978ab; Gonzalez \&Teixeira, 1980).

As minas do Camaquã compreendem dois corpos de minério denominados de Mina Uruguai (minas a céu aberto e subterrânea) e Mina São Luiz (subterrânea) (Fig. 3).0 minério das minas do Camaquã ocorre sob duas formas: em filões e disseminações. Os filões preenchem falhas, apresentando os sulfetos sob forma maciça ou em massas irregulares no interior de ganga de quartzo, hematita, clorita, calcita e barita. A margem dos veios está marcada por intensa alteração hidrotermal representada por silicificação, cloritização, caulinização e sulfetação, ocorrendo quartzo, pirita, calcopirita, bornita, hematita e Au. Calcita e barita representam a mineralogia tardia, ocorrendo disseminadas e como veios com até $15 \mathrm{~cm}$ de espessura nos arenitos e conglomerados 0 minério disseminado ocorre nos conglomerados e arenitos, sendo interpretado por diversos autores como parte das zonas de alteração hidrotermal que se desenvolveram no entorno dos veios, representando a manifestação lateral do controle estrutural da mineralização, com os sulfetos ocupando a porosidade secundária da rocha encaixante. Próximo da superfície há uma zona de oxidação, onde o minério contém pequenas quantidades de ouro e prata. A paragênese é simples, constituída principalmente por sulfetos de cobre: calcopirita, bornita e calcosita, além de pirita (Laux, 1999; Troian, 2009).

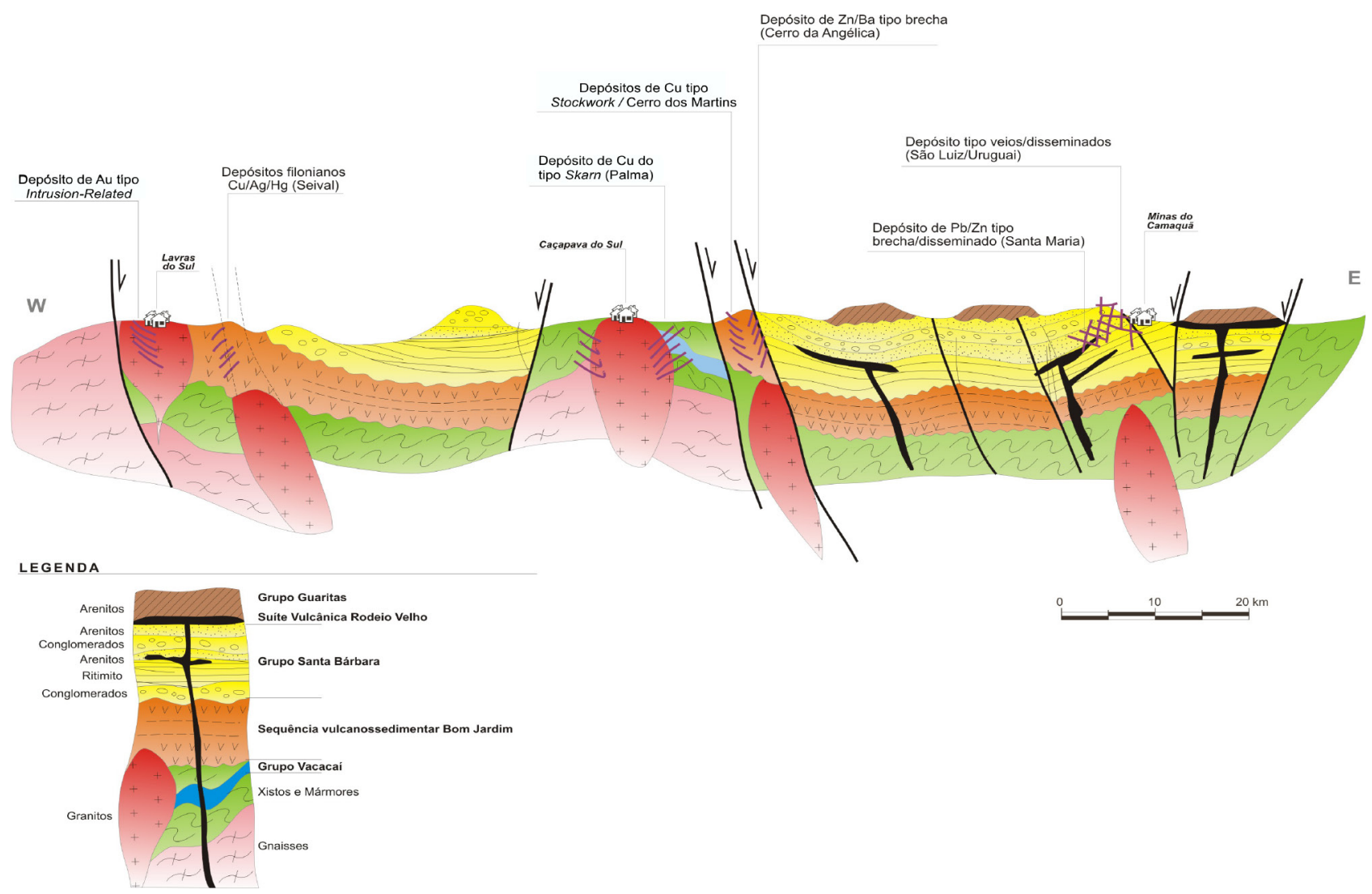

Figura 2. Contexto geológico da área de estudo: Mina Uruguai, minas do Camaquã, Rio Grande do Sul, Brasil (Lago, 2013). 


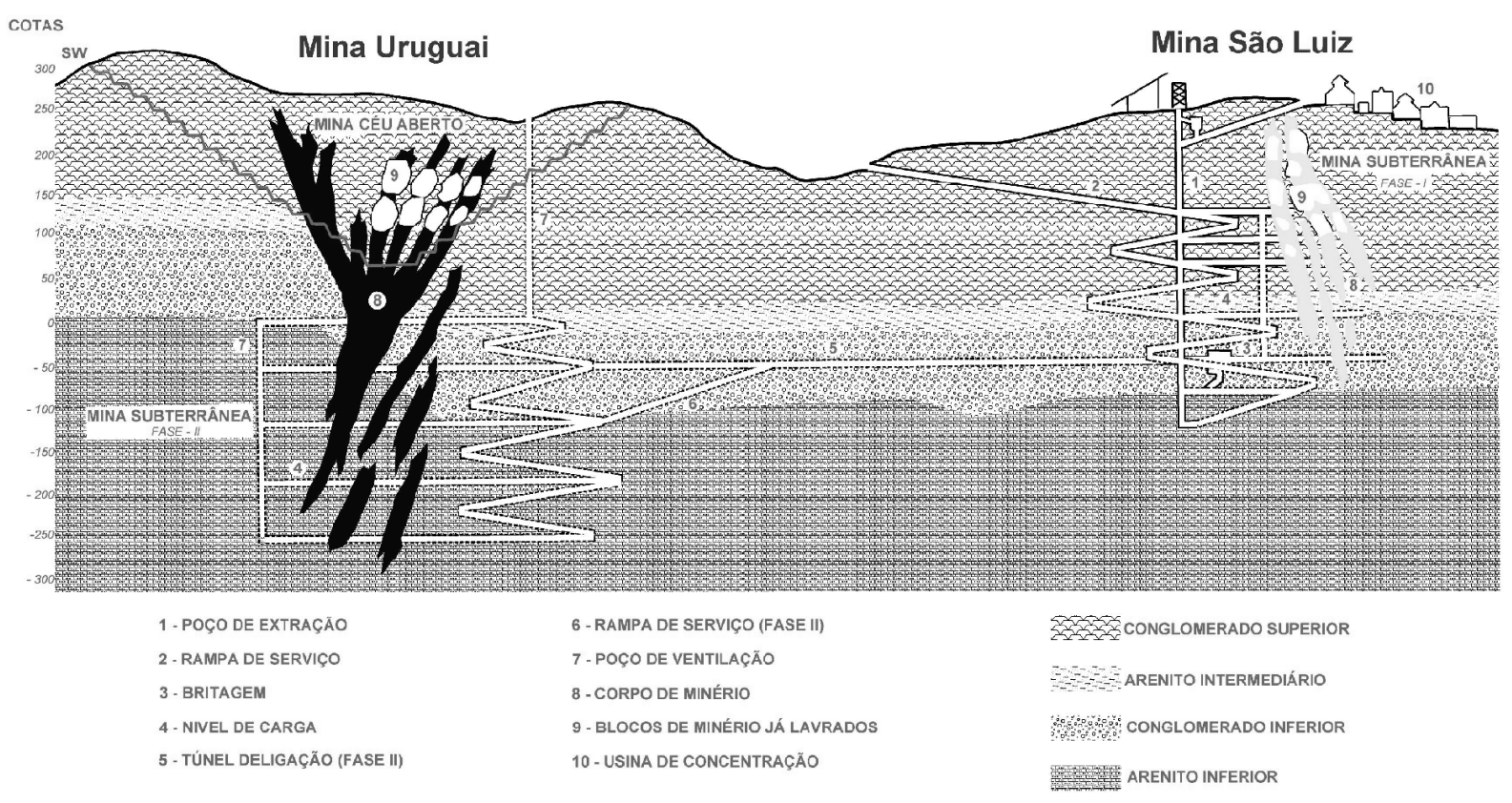

Figura 3. Arranjo geral das instalações das minas Uruguai e Santa Maria, minas do Camaquã, Rio Grande do Sul, Brasil (Lago, 2013).

\subsection{Técnicas e dados utilizados}

\subsubsection{Campanhas de campo}

Na região de estudo foram efetuadas duas campanhas de campo para reconhecimento geo- lógico e coleta de amostras de rocha (tabelas $1 \mathrm{e}$ 2), abrangendo a área das minas do Camaquã e o escritório/depósito da Votorantim Metais, onde estão armazenados os testemunhos de sondagem.

Tabela 1. Relação e características principais de amostras de rocha (afloramentos) utilizadas no estudo, Mina Uruguai, minas do Camaquã, Rio Grande do Sul, Brasil.

\begin{tabular}{ccccc}
\hline Amostra & Latitude & Longitude & Rocha & Alteração \\
\hline $2760-$ RB-R-001 & -30.910575 & -53.443241 & Arenito & Halo de alteração com veios de hematita e \\
barita
\end{tabular}


Tabela 2. Relação e características principais de amostras de rocha (testemunhos de sondagem) utilizadas no estudo, Mina Uruguai, minas do Camaquã, Rio Grande do Sul, Brasil (Fonte: Laux, 1999).

\begin{tabular}{|c|c|c|c|}
\hline Amostra & $\begin{array}{l}\text { Profundidade } \\
\text { (m) }\end{array}$ & Rocha & Alteração \\
\hline U408834-R-001 & 12,12 & $\begin{array}{l}\text { Conglomerado a arenito } \\
\text { conglomerático }\end{array}$ & $\begin{array}{l}\text { Zona de silicificação, com veios e vênulas de } \\
\text { quartzo e calcopirita/pirita }\end{array}$ \\
\hline U408834-R-002 & 16,02 & $\begin{array}{l}\text { Conglomerado a arenito } \\
\text { conglomerático }\end{array}$ & $\begin{array}{l}\text { Zona de silicificação, com veios e vênulas de } \\
\text { quartzo e calcopirita/pirita }\end{array}$ \\
\hline U408834-R-003 & 21,26 & $\begin{array}{l}\text { Conglomerado a arenito } \\
\text { conglomerático }\end{array}$ & $\begin{array}{l}\text { Halo de alteração com veios de quartzo e } \\
\text { hematita }\end{array}$ \\
\hline U408834-R-004 & 27,13 & $\begin{array}{l}\text { Arenito conglomerático a } \\
\text { arenito grosso }\end{array}$ & $\begin{array}{l}\text { Halo de alteração com clorita disseminada junto } \\
\text { com pirita/calcopirita + bornita }\end{array}$ \\
\hline U408834-R-005 & 37,55 & $\begin{array}{l}\text { Arenito conglomerático a } \\
\text { arenito grosso }\end{array}$ & $\begin{array}{l}\text { Zona de brecha (sulfetos e rocha hospedeira } \\
\text { cimentados por quartzo e hematita) }\end{array}$ \\
\hline U408834-R-006 & 38,95 & $\begin{array}{l}\text { Arenito conglomerático a } \\
\text { arenito grosso }\end{array}$ & $\begin{array}{l}\text { Halo de alteração com clorita disseminada junto } \\
\text { com pirita/calcopirita + bornita }\end{array}$ \\
\hline U408834-R-007 & 46,53 & $\begin{array}{l}\text { Arenito conglomerático a } \\
\text { arenito grosso }\end{array}$ & $\begin{array}{l}\text { Halo de alteração com clorita disseminada junto } \\
\text { com pirita/calcopirita + bornita }\end{array}$ \\
\hline U408834-R008 & 50,35 & $\begin{array}{l}\text { Arenito conglomerático a } \\
\text { arenito grosso }\end{array}$ & $\begin{array}{l}\text { Halo de alteração com veios de quartzo e } \\
\text { hematita }\end{array}$ \\
\hline U408834-R-009 & 57,55 & Arenito grosso a arenito médio & $\begin{array}{l}\text { Halo de alteração com veios de quartzo e } \\
\text { hematita }\end{array}$ \\
\hline U7SR0277-R-001 & 74,15 & Arenito grosso a arenito médio & $\begin{array}{l}\text { Halo de alteração com cloritana matriz (pirita / } \\
\text { calcopirita / bornita podem estar associadas) }\end{array}$ \\
\hline U7SR0277-R-002 & 96,08 & $\begin{array}{l}\text { Arenito conglomerático a } \\
\text { arenito grosso }\end{array}$ & $\begin{array}{l}\text { Halo de alteração com clorita disseminada junto } \\
\text { com pirita/calcopirita + bornita }\end{array}$ \\
\hline U7SR0277-R-003 & 120,50 & $\begin{array}{l}\text { Conglomerado a arenito } \\
\text { conglomerático }\end{array}$ & $\begin{array}{l}\text { Halo de alteração com clorita disseminada junto } \\
\text { com pirita/calcopirita + bornita }\end{array}$ \\
\hline U7SR0277-R-004 & 122,10 & $\begin{array}{l}\text { Conglomerado a arenito } \\
\text { conglomerático }\end{array}$ & $\begin{array}{l}\text { Halo de alteração com veios de quartzo e } \\
\text { hematita }\end{array}$ \\
\hline U7SR0277-R-005 & 137,15 & $\begin{array}{l}\text { Conglomerado a arenito } \\
\text { conglomerático }\end{array}$ & $\begin{array}{l}\text { Zona de silicificação, com veios e vênulas de } \\
\text { quartzo e calcopirita/pirita }\end{array}$ \\
\hline U7SR0277-R-006 & 161,40 & $\begin{array}{l}\text { Conglomerado a arenito } \\
\text { conglomerático }\end{array}$ & $\begin{array}{c}\text { Halo de alteração cloríticapervasiva com } \\
\text { pirita + calcopirita disseminadas, cortado por } \\
\text { veios e vênulas de quartzo, calcopirita + pirita } \\
\text { (bornita) }\end{array}$ \\
\hline
\end{tabular}

\subsubsection{Análises espectrais}

As análises espectrais foram realizadas em laboratório, nas dependências da CPRM, em São Paulo, através do espectrorradiômetro ASD-FieldSpec-3 Hi-Resolution com alcance espectral do visível ao infravermelho de ondas curtas (350 a 2500 $\mathrm{nm})$ e resolução espectral de $3 \mathrm{~nm}(\mathrm{em} 700 \mathrm{~nm})$, $8,5 \mathrm{~nm}(\mathrm{em} 1400 \mathrm{n} \mathrm{m})$ e $6,5 \mathrm{~nm}(\mathrm{em} 2500 \mathrm{~nm})$ num total de 2101 bandas. As medidas, registradas em valores de reflectância proporcional relativa a uma referência padrão branca de máxima reflectância difusa (do tipo Spectralon), foram feitas por meio de sonda de contato. Cada amostra foi submetida a várias análises, numa média de 3 , procurando-se medir uma assinatura espectral para cada categoria diferente de face conforme a variação do tipo (exposta, quebra natural ou serrada), grau de intemperismo, cor, relação angular com a foliação ou bandamento principal e relação matriz-cristais, quando pertinente.

A interpretação mineralógica das assinaturas espectrais foi efetuada através da utilização do software The Spectral Geologist (TSG ${ }^{T M}$ Pro), desenvolvido pela Commonwealth Scientific and Industrial Research Organisation (CSIRO), comercializado e distribuído pela AusSpec International, ambas australianas, que permite a discriminação das fases minerais presentes por comparação das feições de absorção no espectro analisado com feições similares de minerais de uma biblioteca espectral de referência. Identificados os minerais presentes, uma desmistura das fases foi calculada, resultando na proporção estimada de cada mineral, e uma curva espectral hipotética foigerada graficamente para comparação com o espectro analisado. Como as análises espectrais são puramente qualitativas, de caráter interpretativo, foram selecionados os 
espectros mais representativos das fases minerais típicas das assembleias de alteração hidrotermal estudadas, que serão discutidos neste artigo.

\section{Resultados}

As assinaturas espectrais medidas nas amostras de rochas sedimentares eopaleozoicas (arenitos e conglomerados afetadas por alteração hidrotermal podem ser visualizadas nas figuras 4, 5 e 6, onde estão ressaltadas as principais feições espectrais (absorção) observadas.

Apesar dos valores de reflectância relativa estarem situados, em sua grande maioria, até 0,3, é possível identificar feições de absorção quando estes espectros são estudados com remoção de contínuo, conforme representado nas curvas espectrais das figuras já citadas. A remoção do contínuo é uma forma de normalizar espectros de reflectância para permitir a comparação de feições de absorção individuais, a partir de uma linha do contínuo comum.

As feições de absorção aparecem no espectro de reflectância como "depressões" e correspondem a regiões do intervalo de comprimentos de onda onde a luz é absorvida devido a ligações moleculares específicas do material alvo. A posição e a profundidade de uma feição, ou razão de profundidades entre duas feições, podem servir para estabelecer a mineralogia das zonas de alteração.
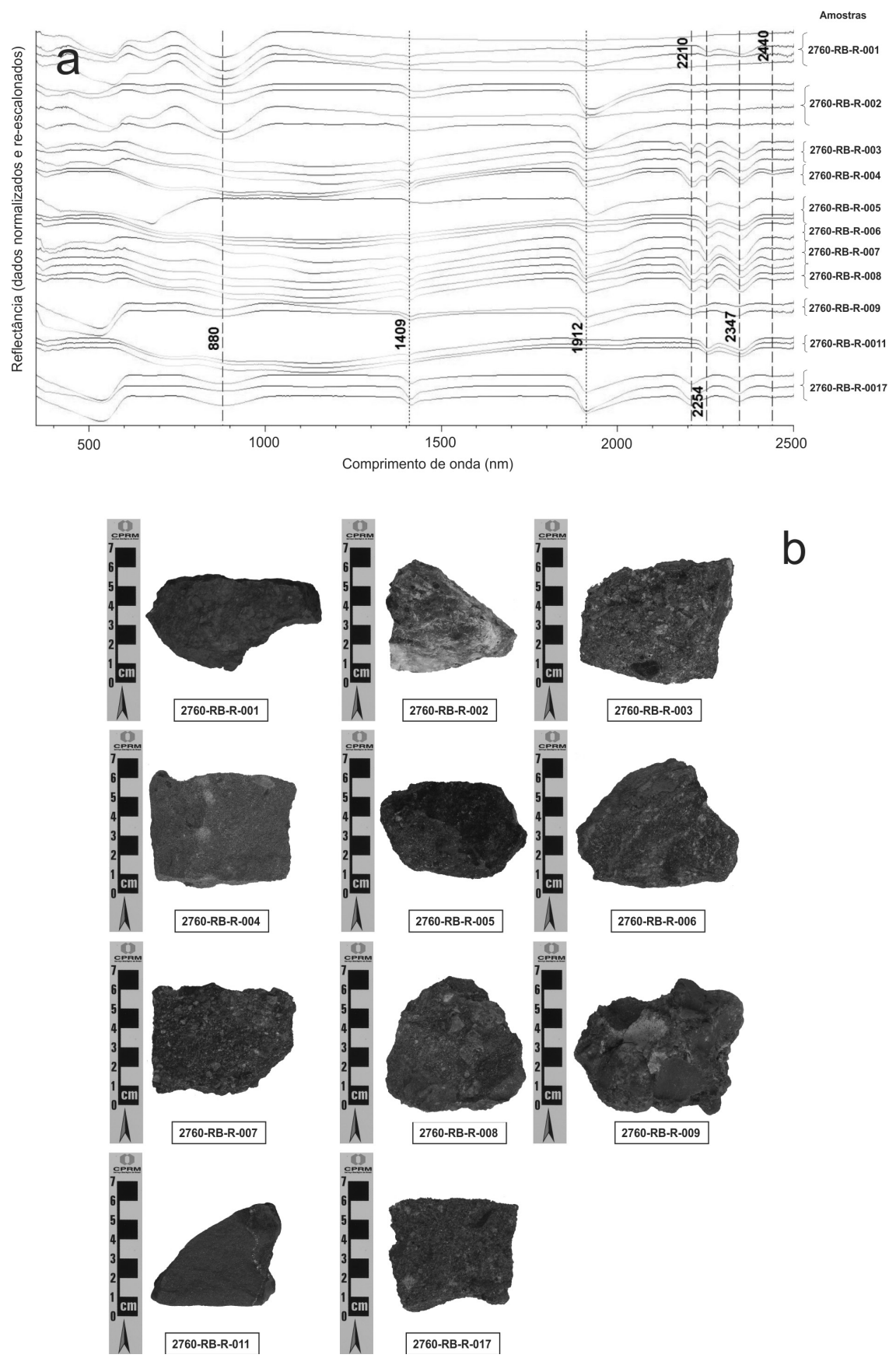

Figura 4. Assinaturas espectrais das amostras de rocha (afloramentos) da área de estudo, minas do Camaquã, Rio Grande do Sul, Brasil. A) Curvas espectrais; B) Amostras de rocha. 

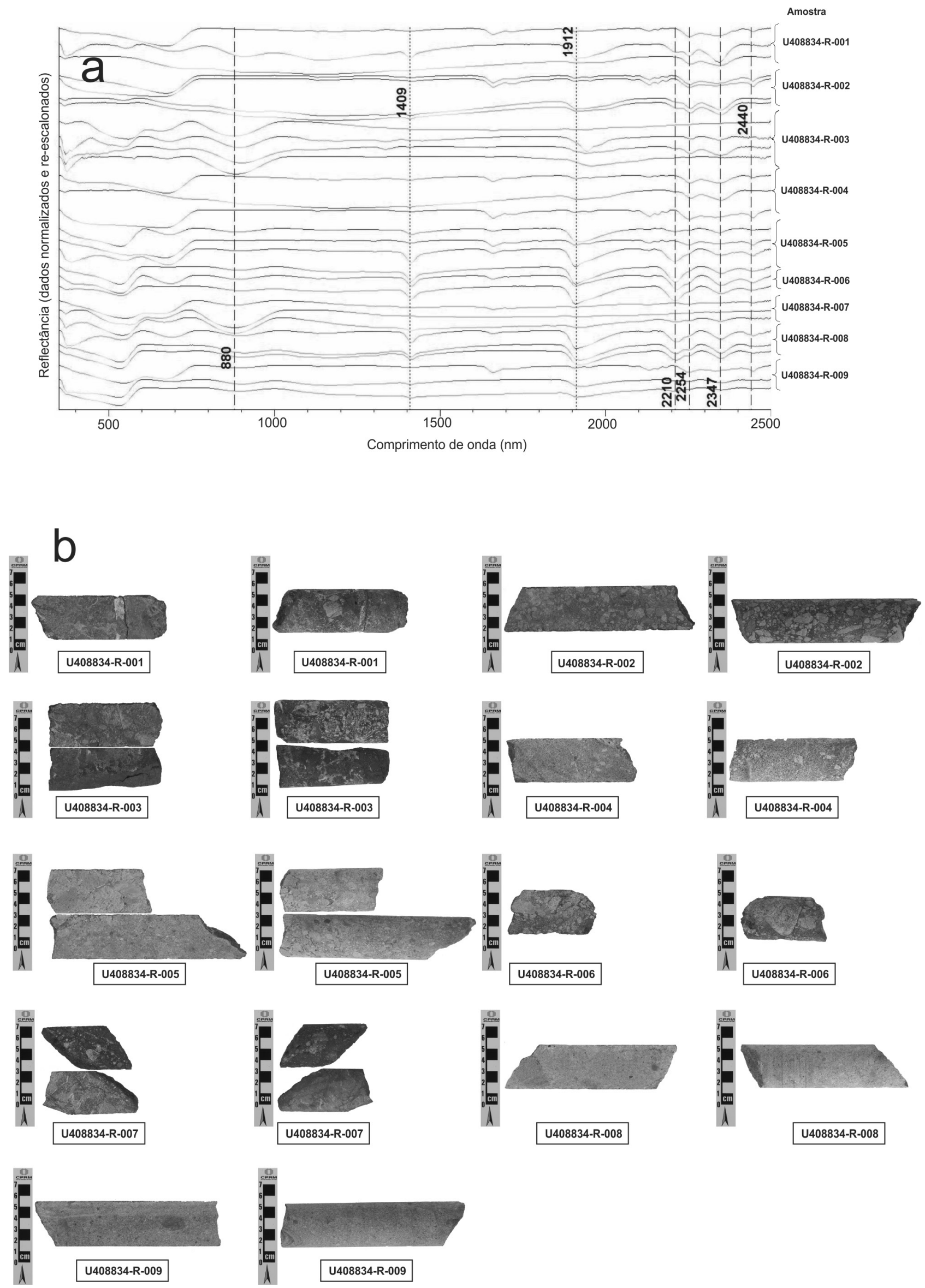

Figura 5. Assinaturas espectrais das amostras de rocha (testemunho de sondagem U408834) da área de estudo, minas do Camaquã, Rio Grande do Sul, Brasil. A) Curvas espectrais; B) Amostras de rocha. 



Figura 6. Assinaturas espectrais das amostras de rocha (testemunho de sondagem U7SR0277) da área de estudo, minas do Camaquã, Rio Grande do Sul, Brasil. A) Curvas espectrais; B) Amostras de rocha.

Com base nas feições de absorção ressaltadas nas curvas espectrais, foram reconhecidos, nas amostras estudadas, os minerais relacionados na sequência (Tab. 3 e 4). Os minerais detectados, no geral gerados em processos metamórfico-hidrotermais mineralizantes, constituem indicadores de vários tipos de depósitos, com destaque para os metálicos, conforme discutido no prosseguimento. 
Tabela 3. Interpretação mineralógica espectral das amostras de rocha (afloramentos) da área de estudo.

\begin{tabular}{|c|c|}
\hline Amostra & Minerais \\
\hline 2760-RB-R-001 & Hematita \\
\hline $2760-R B-R-001$ & Clorita Férrica, Hematita \\
\hline $2760-R B-R-001$ & Clorita Férrica, Hematita \\
\hline $2760-R B-R-002$ & Ilita (Fengita), Hematita \\
\hline $2760-\mathrm{RB}-\mathrm{R}-002$ & Ilita (Fengita), Hematita/Goetita \\
\hline $2760-R B-R-002$ & Ilita (Fengita), Clorita Ferro-Magnesiana, Hematita \\
\hline 2760-RB-R-003 & Ilita (Fengita), Clorita Ferro-Magnesiana, Caulinita \\
\hline $2760-R B-R-003$ & Ilita (Fengita), Clorita Férrica, Goetita \\
\hline $2760-R B-R-004$ & Ilita (Fengita), Clorita Ferro-Magnesiana \\
\hline $2760-R B-R-004$ & Ilita (Fengita), Clorita Ferro-Magnesiana \\
\hline $2760-R B-R-005$ & Clorita Ferro-Magnesiana \\
\hline 2760-RB-R-005 & Clorita Ferro-Magnesiana \\
\hline $2760-R B-R-008$ & Ilita (Fengita), Clorita Ferro-Magnesiana \\
\hline $2760-R B-R-008$ & Ilita (Fengita), Clorita Ferro-Magnesiana, Goetita \\
\hline $2760-R B-R-008$ & Ilita (Fengita), Clorita Ferro-Magnesiana \\
\hline 2760-RB-R-009 & Ilita (Fengita), Hematita \\
\hline $2760-R B-R-009$ & Ilita ("normal" potássica - Muscovita), Clorita Ferro-Magnesiana, Hematita \\
\hline $2760-R B-R-017$ & Ilita (Fengita), Hematita \\
\hline $2760-R B-R-017$ & Ilita (Fengita), Hematita \\
\hline $2760-R B-R-017$ & Ilita (Fengita), Hematita \\
\hline
\end{tabular}

Tabela 4. Interpretação mineralógica espectral das amostras de rocha (testemunhos de sondagem) da área de estudo.

\begin{tabular}{|c|c|c|}
\hline Amostra (Testemunho) & Profundidade (m) & Minerais \\
\hline U408834-R-001 & 12,12 & Clorita Ferro-Magnesiana \\
\hline $\mathrm{U} 408834-\mathrm{R}-001$ & 12,12 & Ilita (Fengita), Clorita Ferro-Magnesiana \\
\hline U408834-R-001 & 12,12 & Clorita Ferro-Magnesiana \\
\hline $\mathrm{U} 408834-\mathrm{R}-002$ & 16,02 & Clorita Ferro-Magnesiana \\
\hline U408834-R-002 & 16,02 & Clorita Ferro-Magnesiana \\
\hline U408834-R-002 & 16,02 & Ilita (Fengita), Clorita Ferro-Magnesiana \\
\hline $\mathrm{U} 408834-\mathrm{R}-002$ & 16,02 & Ilita (Fengita), Clorita Ferro-Magnesiana \\
\hline $\mathrm{U} 408834-\mathrm{R}-003$ & 21,26 & Sulfeto \\
\hline $\mathrm{U} 408834-\mathrm{R}-003$ & 21,26 & Clorita Ferro-Magnesiana \\
\hline $\mathrm{U} 408834-\mathrm{R}-003$ & 21,26 & Ilita (Fengita), Clorita Ferro-Magnesiana \\
\hline $\mathrm{U} 408834-\mathrm{R}-003$ & 21,26 & Hematita \\
\hline U408834-R-004 & 27,13 & Clorita Ferro-Magnesiana \\
\hline $\mathrm{U} 408834-\mathrm{R}-004$ & 27,13 & Ilita (Fengita), Clorita Ferro-Magnesiana \\
\hline $\mathrm{U} 408834-\mathrm{R}-004$ & 27,13 & Clorita Ferro-Magnesiana \\
\hline U408834-R-005 & 37,55 & Ilita (Fengita), Goetita \\
\hline U $408834-\mathrm{R}-005$ & 37,55 & Sulfeto \\
\hline $\mathrm{U} 408834-\mathrm{R}-005$ & 37,55 & Ilita (Fengita) \\
\hline U408834-R-005 & 37,55 & Ilita (Fengita) \\
\hline U408834-R-006 & 38,95 & Ilita (Fengita), Goetita \\
\hline $\mathrm{U} 408834-\mathrm{R}-006$ & 38,95 & Ilita (Fengita), Goetita \\
\hline U408834-R-007 & 46,53 & Hematita \\
\hline $\mathrm{U} 408834-\mathrm{R}-007$ & 46,53 & Hematita \\
\hline $\mathrm{U} 408834-\mathrm{R}-007$ & 46,53 & Ilita (Fengita), Clorita Ferro-Magnesiana, Hematita \\
\hline $\mathrm{U} 408834-\mathrm{R}-008$ & 50,35 & Ilita (Fengita) \\
\hline $\mathrm{U} 408834-\mathrm{R}-008$ & 50,35 & Ilita (Fengita), Clorita Ferro-Magnesiana \\
\hline U408834-R-008 & 50,35 & Ilita (Fengita), Clorita Ferro-Magnesiana \\
\hline $\mathrm{U} 408834-\mathrm{R}-009$ & 57,55 & Hematita/Goetita \\
\hline U408834-R-009 & 57,55 & Ilita (Fengita), Clorita Ferro-Magnesiana, Hematita/Goetita \\
\hline $\mathrm{U} 408834-\mathrm{R}-009$ & 57,55 & Ilita (Fengita), Clorita Ferro-Magnesiana, Hematita/Goetita \\
\hline U7SR0277-R-001 & 74,15 & Ilita (Fengita), Clorita Ferro-Magnesiana, Hematita/Goetita \\
\hline U7SR0277-R-001 & 74,15 & Ilita (Fengita), Clorita Ferro-Magnesiana, Goetita \\
\hline U7SR0277-R-001 & 74.15 & Clorita Ferro-Magnesiana, Goetita \\
\hline U7SR0277-R-002 & 96,08 & Ilita (Fengita), Clorita Ferro-Magnesiana, Hematita \\
\hline U7SR0277-R-002 & 96,08 & Sulfeto \\
\hline U7SR0277-R-002 & 96,08 & Clorita Ferro-Magnesiana, Goetita \\
\hline U7SR0277-R-002 & 96,08 & Ilita (Fengita), Clorita Ferro-Magnesiana, Goetita \\
\hline U7SR0277-R-003 & 120,5 & Clorita Ferro-Magnesiana \\
\hline U7SR0277-R-003 & 120,5 & Clorita Ferro-Magnesiana \\
\hline U7SR0277-R-003 & 120,5 & Clorita Ferro-Magnesiana \\
\hline U7SR0277-R-004 & 122,1 & Clorita Ferro-Magnesiana, Goetita \\
\hline U7SR0277-R-004 & 122,1 & Hematita \\
\hline U7SR0277-R-005 & 137,15 & Clorita Ferro-Magnesiana \\
\hline U7SR0277-R-005 & 137,15 & Ilita ("normal" potássica - Muscovita), Clorita Ferro-Magnesiana \\
\hline U7SR0277-R-006 & 161,4 & Clorita Ferro-Magnesiana, Goetita \\
\hline U7SR0277-R-006 & 161,4 & Ilita (Fengita), Clorita Ferro-Magnesiana, Goetita \\
\hline
\end{tabular}




\section{Discussão dos resultados}

A posição, forma, profundidade e largura das feições de absorção são controladas pela estrutura cristalina e composição química dos diferentes minerais, produzidas como consequência de processos eletrônicos (transições atômicas) e vibracionais (transições moleculares) (Hunt, 1977).

Na grande maioria, as análises espectrais das rochas afetadas em graus variáveis por alteração tanto hidrotermal como intempérica, resultam em espectros mistos da combinação de diversos minerais. Feições espectrais diagnósticas normalmente são preservadas nestas misturas de forma que é possível identificar as fases minerais envolvidas. Há que se observar que as feições das curvas espectrais das amostras estudadas geralmente não se ajustam perfeitamente às da biblioteca espectral de referência, tendo em vista a presença de misturas minerais, diferenças na composição geoquímica ou no grau de ordenamento cristalino dos minerais.

Da análise espectral das amostras da área de estudo, puderam ser identificados os seguintes minerais principais: caulinita, ilita (tendendo para fengita ou muscovita), clorita (férrica e ferro-magnesiana), hematita e goetita.

Para micas brancas e argilas, os picos das feições de absorção em 2200 nm (ligação Al-OH) e em 1910 (água molecular) são críticos para sua identificação. Para cloritas, o pico da feição de absorção em $\sim 2250 \mathrm{~nm}$ (ligação Fe-OH) e em $\sim 2350$ nm (ligação $\mathrm{Mg}-\mathrm{OH}$ ) e a forma do espectro entre 1400 e $1900 \mathrm{~nm}$ têm sido utilizados para identificar o conteúdo de Fe:Mg (Clark et al., 2007; Cohen, 2011).

A caulinita apresenta feições de absorção duplas localizadas entorno de $1400 \mathrm{~nm}$ e $2200 \mathrm{~nm}$. A feição dupla em 1400 nm é função das vibrações nos íons hidroxilas ( $\mathrm{OH}-)$ e moléculas de água (H20), enquanto a feição dupla em $\sim 2200 \mathrm{~nm}$ deve-se à energia vibracional da ligação Al-OH. A presença da feição de absorção em 1900 nm indica presença de água molecular na amostra. Em amostras com alto conteúdo de sílica ou baixas concentrações de caulinita, a feição da ligação $\mathrm{Al}-\mathrm{OH}$ pode estar modificada ou decrescer para uma pequena inflexão (Harraden et al., 2013).

A ilita apresenta cinco feições de absorção principais: $1400 \mathrm{~nm}$ (vibrações de íons hidroxila $\mathrm{OH}-$ ), $1900 \mathrm{~nm}$ (água molecular), $2200 \mathrm{~nm}$ (diagnóstica da ilita e variável segundo a sua composição), $\sim 2347 \mathrm{~nm}$ e $\sim 2440 \mathrm{~nm}$ (diagnósticas da ilita e consistentes mesmo em casos de misturas da ilita com outros minerais). Foi identificado o pre- domínio, em termos composicionais, de ilita rica em Mg-Fe (tendendo para fengita) e, em poucas amostras, de ilita rica em $\mathrm{K}$ (tendendo para muscovita), observando-se uma tendência de aumento progressivo do comprimento de onda da feição de absorção principal dos termos ricos em potássio até os enriquecidos em ferro/magnésio.

A paleotemperatura de cristalização da clorita, refletida em sua composição química, mostra particular interesse para os estudos de gênese dos depósitos minerais. A clorita apresenta feições de absorção diagnósticas em 2235-2255 $\mathrm{nm}$ e $2320-2360 \mathrm{~nm}$, dependendo da relação $\mathrm{Fe} /$ Mg (GMEX, 2008). Nas amostras estudadas foram identificadas cloritas férricas e ferro-magnesianas, em conformidade com Troian (2009). Para este autor, a clorita é o argilomineral mais abundante nas rochas afetadas pela alteração hidrotermal na Mina Uruguai, ocorrendo alterando a matriz da rocha encaixante das mineralizações; como veios/ vênulas com ilita, sulfetos (pirita e calcopirita) e hematita venular associados; e alterando minerais detríticos, principalmente a biotita. A variação na quantidade de ferro nas cloritas geradas por processos hidrotermais indica a ocorrência de pelo menos dois pulsos no processo de alteração hidrotermal.

Os óxidos de ferro produzem feições de absorção características devido aos processos eletrônicos envolvidos nas ligações do $\mathrm{Fe}^{3+}$ com o oxigênio (hematita) ou com oxigênio e hidroxila (goetita) (Hunt \& Ashley,1979; Cudahy et al., 2008). A goetita e a hematita apresentam feições de absorção características entre 650 e $715 \mathrm{~nm}$ e entre 850 e $1000 \mathrm{~nm}$ (Morris, 1985).

A paragênese identificada, constituída por caulinita + ilita (fengita + muscovita) + clorita (férrica e ferromagnesiana) + hematita + goetita, associada a outras características observadas na Mina Uruguai, é consistente com alteração hidrotermal sob condições epitermais de baixo enxofre, num subtipo apresentado por Sillitoe (1993), como proposto em Laux et al. (2005).

Os depósitos epitermais se formam no intervalo de temperaturas de $<150^{\circ} \mathrm{C} \mathrm{a} \sim 300^{\circ} \mathrm{C}$, em condições crustais que vão da superfície até uma profundidade de 1 a $2 \mathrm{~km}$, tendo como características diagnósticas altos conteúdos de $\mathrm{Au}$ e $\mathrm{Ag}$, baixa salinidade (frequentemente $<5 \%$ equiv. peso de $\mathrm{NaCl}$ ), evidência de boiling (ebulição) e de água meteórica como a maior fonte dos fluidos mineralizantes (White \& Hedenquist, 1995).

Tais depósitos podem ser divididos em dois tipos principais, de acordo com as assembleias minerais características do minério, da ganga e das 
alterações hidrotermais: alto enxofre (ácido-sulfato ou alunita-caulinita \pm pirofilita) e baixo enxofre (adulária-sericita \pm ilita é o tipo mais comum) (Hayba et al., 1985; Berger \& Heley, 1988). Além da paragênese, os depósitos epitermais de baixo enxofre diferem dos de alta sulfetação por apresentarem uma estrutura de veios melhor definida, menor afinidade magmática, por serem gerados a partir de fluidos com menores temperaturas (100$\left.250^{\circ} \mathrm{C}\right)$, salinidades atenuadas $(0-3 \% \mathrm{de} \mathrm{NaCl}), \mathrm{pH}$ neutro, e em ambiente de deposição redutor (Marcoux, 1995).

Na Mina Uruguai, a alteração hidrotermal ocorre nas proximidades das zonas de fratura e filões, sob a forma de cloritização, silicificação e sericitização, se estendendo a partir dos filões apenas por alguns metros, consistente com o modelo do tipo epitermal, diferentemente do que ocorre, por exemplo, nos depósitos de cobre pórfiro, onde ela é intensa e pervasiva. As temperaturas, entre 187 e $328^{\circ} \mathrm{C}$ (Lima, 1998; Laux \& Lindenmayer, 1998; Ronchi et al., 1998) e as baixas a moderadas salinidades dos fluidos (Beckel, 1990; Bettencourt, 1976), até $14 \%$ equiv. peso de $\mathrm{NaCl}$, estimadas nas Minas do Camaquã, também estão de acordo com o modelo epitermal.

\section{Conclusões}

No estudo e definição de um sistema epitermal, a identificação dos principais minerais constituintes e do zoneamento das assembleias de alteração hidrotermal são características distintivas fundamentais.

A espectroscopia de reflectância mostrou-se uma ferramenta adequada para a identificação dos minerais de alteração das rochas estudadas, tendo corroborado os estudos geológicos prévios realizados na área de estudo.

A rapidez com que são obtidos os resultados e a característica não destrutiva do método utilizado validam a utilização desta técnica em programas de exploração mineral, especialmente nas fases iniciais, mesmo em áreas com condições fisiográficas pouco favoráveis, de clima úmido, com cobertura vegetal significativa e alteração intempérica.

A paragênese identificada, constituída por caulinita + ilita (fengita + muscovita) + clorita (férrica e ferromagnesiana) + hematita + goetita, associada a outras características observadas na Mina Uruguai, é consistente com alteração hidrotermal sob condições epitermais de baixo enxofre.

As curvas espectrais obtidas serão incorporadas à biblioteca espectral do Serviço Geológico do Brasil (CPRM) para aplicações futuras na própria área de estudo ou em áreas de contexto geológico/ metalogenético similares.

As assinaturas minerais identificadas mostram-se consistentes como membros finais de referência no mapeamento espectral aplicado a dados do sensor ASTER (Advanced Spaceborne Thermal Emission and Reflection Radiometer), em etapa futura, validando o potencial dos dados em investigações geológicas e de exploração mineral.

\section{Referências}

Beckel, J. 1990. Metalogenia del Cu, Pb y Zn em la Cuenca de Camaquã Durante el Ciclo Orogenico Brasiliano, Rio Grande del Sur (Brasil). Salamanca. 275p. Tese de Doutorado, Universidade de Salamanca.

Berger, B.B. \& Heley, R.W. 1988. Advances in understanding of epithermal gold-silver deposits, with special reference to the Western United. Economic Geology, 6: 405-423.

Bergmann, M., Hoff, R. \& Ducati, J.R. 2008. Espectrorradiometria para validação de dados geológicos e de solos como contribuição ao estabelecimento de terroirs na Metade Sul (RS, Brasil). In: CONGRESSO BRASILEIRO DE GEOLOGIA, 44., 2008, Curitiba. Anais... Curitiba, SBG, v.1, p. 599.

Bergmann, M., Hoff, R. \& Ducati, J.R. 2009. Implantação da biblioteca espectral de rochas e solos da CPRM: um resgate da memória espectral da litoteca e apoio a Projetos de Levantamentos Geológicos Básicos (PLGB). In: SIMPÓSIO BRASILEIRO DE SENSORIAMENTO REMOTO, 14., 2009, Natal. Anais... Natal, INPE, v.1. p. 1-8.

Bettencourt, J.S. 1972. A mina de cobre de Camaquã, Rio Grande do Sul. São Paulo, 175p. Tese de Doutorado, Instituto de Geociências, Universidade de São Paulo.

Bettencourt, J.S. 1976. Mineralogie, inclusions fluides et isotopes stables d'oxigène et de soufre de la mine de culvre de Camaquã - RS, (une étude preliminaire). In: CONGRESSO BRASILEIRO DE GEOLOGIA, 29., Ouro Preto, 1976. Anais... Ouro Preto, SBG, v.2, p. 409-423.

Clark, R.N., Swayze, G.A., Wise, R., Livo, E., Hoefen, T., Kokaly, R. \& Sutley, S.J. 2007. USGS digital spectral library splib06a. (U.S. Geological Survey, Digital Data Series 231).

Cohen, J.F. 2011. Compositional Variations in Hydrothermal White Mica and Chlorite from Wall-Rock Alteration at the Ann-Mason Porphyry Copper Deposit, Nevada. Corvallis, 111p. Master of Science in Geology, Oregon State University.

Cudahy, T., Jones, M., Thomas, M., Laukamp, C., Caccetta, M., Hewson R., Rodger A. \& Verrall, M. 2008. Next generation mineral mapping: Queensland airborne HyMap and satellite ASTER surveys 2006-2008. 161 p. (CSIRO report P2007/364).

Cudahy, T., Hewson, R., Caccetta, M., Roache, A., Whitbourn, L., Connor, P., Coward, D., Mason, P., Yang, K., Huntington, J. \& Quigley, M. 2009. Drill core logging of Plagioclase feldspar composition and other min- 
erals associated with Archean gold mineralization at Kambalda, Western Australia, using a bidirectional thermal infrared reflectance system. Reviews in Economic Geology, 16: 223-235.

Doublier, M.P., Roache, A. \& Potel, S. 2010. Short wavelength infrared spectroscopy: a new petrological tool in low- to very low grade pelites. Geology, 38: 1031-1034.

Fambrini, G.L. 2003. O Grupo Santa Bárbara (Neoproterozóico III) a norte do rio Camaquã, Rio Grande do Sul, Brasil. São Paulo, 243p. Tese de Doutorado, Programa de Pós-graduação em Geoquímica e Geotectônica, Instituto de Geociências, Universidade de São Paulo.

GMEX. 2008. The spectral interpretation field manual, 3.ed. Sidney, AusSpec International Ltd., $189 \mathrm{p}$

Gonzalez, M.A. \& Teixeira, G. 1980. Considerações sobre a estratigrafia e ambientes de sedimentação da região das Minas do Camaquã e Jazida Santa Maria RS. In: CONGRESSO BRASILEIRO DE GEOLOGIA, 31. 1980, Balneário Camboriu. Anais... Balneário Camboriu, SBG, v.3, p. 1513-1524.

Harraden, C.L., Mcnulty, B.A., Gregory, M.J. \& Lang, J.R. 2013. Short wave infrared spectral analysis of hydrothermal alteration associated with the pebble porphyry copper-gold-molybdenum deposit, Iliamna, Alaska. Economic Geology, 108: 483-494.

Hayba, D.O., Bethke, P.M., Heald, P. \& Foley, N.K. 1985. Geologic, mineralogical, and geochemical characteristics of volcanic-hosted epithermal precious-metal deposits. Reviews in Economic Geology, 5: 129-162.

Herrmann, W., Blake, M., Doyle, M., Huston, D., Kamprad, J., Merry, N. \& Pontual, S. 2001. Short wavelength infrared (SWIR) spectral analysis of hydrothermal alteration zones associated with base metal sulfide deposits at Rosebery and Western Tharsis, Tasmania, and Highway-Reward, Queensland. Economic Geology, 96: 939-955.

Herrmann, W., Green, G.R., Barton, M.D. \& Davidson, G.J. 2009. Litho geochemical and stable isotopic insights into submarine genesis of pyrophyllite-altered facies at the Boco Prospect, western Tasmania. Economic Geology, 104(6): 775.

Hook S.J., Karlstrom K.E., Miller C.F. \& Mccaffrey, K.J.W. 1994. Mapping the Piute Mountains, California, with thermal infrared multispectral scanner (TIMS) images. Journal of Geophysical Research Solid Earth (1978-2012), 99 (B8): 15605-15622.

Hunt, G. 1977. Spectral signatures of particulate minerals in the visible and near infrared. Geophysics, 42(3): 501-513.

Hunt, G.R. \& Ashley, R.P. 1979. Spectra of altered rocks in the visible and near infrared. Economic Geology, 74(7): 1613.

Jones, S., Herrmann, W. \& Gemmell, J.B. 2005. Short wave length infrared spectral characteristics of the HW horizon: implications for exploration in themyra falls volcanic-hosted massive sulfide camp, Vancouver Island, British Columbia, Canada. Economic Geology, 100(2): 273.

Justo, A.P., Perrotta, M.M., Souza Filho, C.R , Senna, J.A.
\& Quitete, E.B. 2012. Espectroscopia de reflectância de minerais de pegmatitos e suas encaixantes: primeiros dados do distrito pegmatítico Solonópole-Banabuiú. In: CONGRESSO BRASILEIRO DE GEOLOGIA, 46., 2012, Santos, SP. Anais... Santos, SP, SBG. 1 CD-ROM. PAP011394.

Justo, A.P., Bergmann, M. \& Perrotta, M.M. 2013. Identificação de Minerais do Grupo das Zeólitas por Espectroscopia de Reflectância visando à Remineralização de Solos para Agricultura. In: CONGRESSO BRASILEIRO DE ROCHAGEM, 2., 2013, Poços de Caldas, MG. Anais... Poços de Caldas, MG, PETROBRAS/EMBRAPA, p. 265-274.

Lago, S. 2013. Síntese geológica do depósito de Zn-Pb de Santa Maria-RS: Votorantim Metais. In: SIMPÓSIO BRASILEIRO DE METALOGENIA, 3., 2013, Gramado. Palestra técnica. Gramado, UFRGS.

Laukamp, C., Cudahy, T., Thomas, M., Jones, M., Cleverley, J.S. \& Oliver, N.H.S. 2011. Hydrothermal mineral alteration patterns in the Mount Isa Inlier revealed by airborne hyperspectral data, Australian Journal of Earth Sciences, 58: 1-20.

Laux, J.H. 1999. Caracterização da mineralização cupro-aurífera de uma parte da Mina Uruguai, Caçapava do Sul-RS. São Leopoldo, RS. 135p. Dissertação de Mestrado, Programa de Pós-graduação em Geologia, Universidade do Vale do Rio dos Sinos.

Laux, J.H. \& Lindenmayer, Z.G. 1998. Caracterização da mineralização $\mathrm{Cu}(\mathrm{Au})$ de um setor da Mina Uruguai, Minas do Camaquã, Brasil: Geotermometria da clorita. In: CONGRESSO LATINOAMERICANO DE GEOLOGIA, 10., 1998, Buenos Aires. Actas... Buenos Aires, SEGEMAR, v. 3, p. 124-129.

Laux, J.H., Lindenmayer, Z., Teixeira, J. \& Bastos Neto, A. 2005. Ore genesis at the Camaquã copper mine, a Neoproterozoic sediment-hosted deposit in Southern Brazil. Ore Geology Reviews, 26(1): 71-89.

Lima, L. de. 1998. A Mina Uruguai e Jazida Santa Maria, distrito mineiro de Camaquã (RS): um estudo petrológico, geoquímico e geotermométrico. São Leopoldo, RS. 109p. Dissertação de Mestrado, Programa de Pós-graduação em Geologia, Universidade do Vale do Rio dos Sinos.

Marcoux, E. 1995. Gold and volcanoes: epithermal gold deposits, a review. Comptes Rendus de l'Académie des Sciences Paris, 321(2A): 723-735.

Morris, R.C. 1985. Genesis of iron ore in banded iron-formation by supergene and supergene-metamorphic processes, a conceptual model. In: Wolf, K.H. (ed.), Handbook of Strata-Bound and Stratiform Ore Deposits, Amsterdam, Elsevier, v. 13, p. 73-235.

Paim, P.S.G., Faccini, U.F., Guimarães Netto, R. \& Nowatzki, C.H. 1992. Estratigrafia de seqüências e sistemas deposicionais das bacias do Camaquã e Santa Bárbara, Eo-paleozóico do RS (Brasil). Correlación Geologica, 9: 41-45.

Perrotta, M.M. 2004. Assinatura espectral de intrusões félsicas em imagens ASTER. In: CONGRESSO BRASILEIRO DE GEOLOGIA, 42., 2004, Araxá, MG. Anais... Araxá, MG, SBG. 1 CD-ROM.

Perrotta, M.M., Souza Filho, C.R. \& Leite, C.A.S. 2005. Ma- 
peamento espectral de intrusões pegmatíticas relacionadas a mineralizações de lítio, gemas e minerais industriais na região do Vale do Jequitinhonha (MG) a partir de imagens ASTER. In: SIMPÓSIO BRASILEIRO DE SENSORIAMENTO REMOTO, 12., 2005, Goiânia. Anais... Goiânia, INPE, p. 1855-1862.

Perrotta, M.M., Justo, A. P., Souza Filho, C. R., Senna, J. A. \& Quitete, E. B. 2012. Biblioteca Espectral do Serviço Geológico do Brasil - ensaio de aplicação: rochas metavulcânicas do Supergrupo Espinhaço Oriental, Bahia, PAP01053. In: CONGRESSO BRASILEIRO DE GEOLOGIA, 46., 2012, Santos, SP. Anais... Santos, SP, SBG. 1 CD-ROM.

Perrotta, M.M., Justo, A.P., Souza Filho, C.R., Senna, J.A., Almeida, T.A.S. \& Oliveira, S.A.M. 2013. Estudo de assinaturas espectrais de metavulcânicas do Espinhaço Setentrional e sua aplicação no mapeamento mineral em imagem do sensor ASTER: dados da Biblioteca Espectral do Serviço Geológico do Brasil. In: SIMPÓSIO BRASILEIRO DE SENSORIAMENTO REMOTO, 16., 2013, Foz do Iguaçu. Anais... Foz do Iguaçu, MCT/INPE. p. 3656-3663.

Perrotta, M.M., Martins, A.A.M., Almeida, R.C., Melo, T.G.G., Oliveira, S.A.M. \& Costa, M.A.C. 2014. Dados de Aerolevantamentos por Sensor Hiperespectral: ensaio de classificação mineralógica metavulcânicas do espinhaço setentrional, Bahia, In: CONGRESSO BRASILEIRO DE GEOLOGIA, 47., 2014, Salvador. Anais... Salvador, SBG. 1 CD ROM. PAP016465.

Ribeiro, M., Bocchi, P.R., Figueiredo Filho, P. M. \& Tessari, R.J. 1966. Geologia da Quadrícula de Caçapava do Sul, RS. Boletim da Divisão de Fomento da Produção Mineral, DNPM, Rio de Janeiro, 232p.

Ronchi, L.H., Murta, C.R. \& Godoy, M.M. 1998. O minério sulfetado no Arenito Inferior da Mina de Cobre Uruguai, RS. In: CONGRESSO BRASILEIRO DE GEOLOGIA, 40., 1998, Belo Horizonte, Resumos... Belo Horizonte, SBG. p. 154.

Sillitoe, R.H. 1993. Epithermal models: Genetic types, geometrical controls and shallow features. Mineral

Manuscrito 562.

Editores: Maria do Carmo Lima e Cunha e Paulo A.

Souza.
Deposit Modeling, 40: 403-417.

Sonntag, I., Laukamp, C. \& Hagemann, S.G. 2012. Low potassium hydrothermal alteration in low sulfidation epithermal systems as detected by IRS and XRD: Na example from the Co-0 mine, Eastern Mindanao, Philippines. Ore Geology Reviews, 45: 47-60.

Sun, Y., Seccombe, P. K., \& Yang, K. 2001. Application of short-wave infrared spectroscopy to define alteration zones associated with the Elurazinc-lead-silver deposit, NSW, Australia. Journal of Geochemical Exploration, 73(1): 11-26.

Teixeira, G., Gonzales, A.P., Gonzales, M.A. \& Licht, O.A.B. 1978a. Contribuição ao estudo de mineralizações cupríferas disseminadas no Distrito Minas do Camaquã. In: CONGRESSO BRASILEIRO DE GEOLOGIA, 30., 1978, Recife. Anais. . Recife, SBG. p. 1644-1654.

Teixeira, G., Gonzales, A.P., Gonzales, M.A. \& Licht, O.A.B. 1978b. Situação das Minas do Camaquã, Rio Grande do Sul. In: CONGRESSO BRASILEIRO DE GEOLOGIA, 30., 1978, Recife. Anais... Recife, SBG. p. 1893-1905.

Troian, G. C. 2009. A cloritização na Mina Uruguai, Minas do Camaquã, RS/Brasil. Porto Alegre, 87p. Dissertação de Mestrado, Programa de Pós-graduação em Geociências, Instituto de Geociências, Universidade Federal do Rio Grande do Sul.

White, N.C. \& Hedenquist, J.W. 1995. Epithermal gold deposits: Styles, characteristics and exploration. $S E G$ Newsletter, 23(1): 9-13.

Yang K., Lian C., Huntington J. F., Peng, Q. \& Wang, Q. 2005. Infrared spectral reflectance characterization of the hydrothermal alteration at the Tuwu $\mathrm{Cu}-\mathrm{Au}$ deposit, Xinjiang, China. Mineralium Deposita, 40: 324-336.

Yang, K., Browne, P., Huntington, J. \& Walshe, J. 2001. Characterizing the hydrothermal alteration of the Broadlands-Ohaaki geothermal system, New Zealand, using short-wave infrared spectroscopy. Journal of Volcanology and Geothermal Research, 106(12): 53-65. 